\title{
Spindle Cell Melanoma: A Comparison of the Cytomorphological Features with the Epithelioid Variant
}

\author{
Ritika Walia Deepali Jain Sandeep R. Mathur Venkateswaran K. Iyer \\ Department of Pathology, All India Institute of Medical Sciences, New Delhi, India
}

\section{Key Words}

Spindle cell melanoma $\cdot$ Cytology $\cdot$ Metastasis

\begin{abstract}
Objectives: Fine-needle aspiration cytology serves as a rapid and cost-effective tool for the diagnosis of melanoma, especially in the recurrent and metastatic cases. The diagnosis poses a challenge due to the varied morphological patterns. Spindle cell melanoma mimics other sarcomas and carcinomas on morphology. This study highlights the cytomorphological features of spindle cell melanoma and compares them with the conventional epithelioid type. Study Design: Cytology smears of 55 aspirates from 45 diagnosed cases of melanoma from various sites were reviewed. Histopathology correlation was done in spindle and mixed cell tumors. Results: Melanomas with a pure or mixed spindle cell component occurred in $31 \%$ of the cases and in a slightly higher age group. These demonstrated prominent cellular cohesion ( $p<0.0001)$, mild to moderate nuclear atypia and inconspicuous to small nucleoli as compared to the epithelioid variant. The presence of melanin pigment was a useful clue to the diagnosis. Most of the cases correlated well with the histomorphology. Conclusion: Spindle cell melanoma is a morphological variant which can be readily misinterpreted due to a lack of classical cytological features of melanoma.
\end{abstract}

Hence, these are vulnerable to be misinterpreted as other neoplasms. An awareness of clinical and cytological features is important to reach the correct diagnosis.

(C) 2013 S. Karger AG, Basel

\section{Introduction}

Melanoma is an aggressive tumor and has a propensity to metastasize to lymph nodes, lungs, liver and virtually any other site of the body $[1,2]$. An early and accurate diagnosis of the tumor at the site of recurrence or metastasis is necessary owing to the poor prognosis of the tumor. Fine-needle aspiration (FNA) cytology with or without image guidance serves as an excellent tool for accurate diagnosis, and hence early recognition of metastatic and recurrent cases is possible. Morphologically, melanoma is known to mimic epithelial neoplasms, sarcomas and lymphomas on histopathology as well as on cytological smears [3-6]. Earlier studies have described variable cytomorphological features of melanomas in FNA smears [4-6]. The spindle cell type is a rare variant with an incidence of $3-14 \%$ in different series $[6,7]$. Detailed cytomorphological analysis of the spindle cell variant has rarely been described [5], and a comparison with the conventional epithelioid type has not been performed to the

\section{KARGER}

E-Mail karger@karger.com

www.karger.com/acy
(C) 2013 S. Karger AG, Basel

0001-5547/13/0576-0557\$38.00/0
Correspondence to: Dr. Deepali Jain

Department of Pathology

All India Institute of Medical Sciences

Ansari Nagar, New Delhi 110029 (India)

E-Mail deepalijain76@gmail.com 
Table 1. Comparative analysis of spindle and epithelioid variants

\begin{tabular}{|c|c|c|c|c|c|c|c|c|c|}
\hline \multirow[t]{2}{*}{ Cell type } & \multicolumn{2}{|c|}{ Architecture } & \multirow[t]{2}{*}{ Melanin } & \multicolumn{3}{|c|}{ Nuclear pleomorphism } & \multicolumn{3}{|l|}{ Nucleoli } \\
\hline & dyscohesive & cohesive & & mild & moderate & marked & inconspicuous & prominent & macro nucleoli \\
\hline Mixed cells $(n=8)$ & 0 & 8 & 6 & 1 & 4 & 3 & 4 & 3 & 1 \\
\hline Spindle and mixed cells $(\mathrm{n}=14)$ & 1 & 13 & 11 & 3 & 8 & 3 & 8 & 5 & 1 \\
\hline Epithelioid type $(\mathrm{n}=31)$ & 27 & 4 & 25 & 7 & 16 & 8 & 5 & 11 & 15 \\
\hline
\end{tabular}

best of our knowledge. This study was conducted with the objective of documenting the cytological features of spindle cell melanoma and comparing them with the epithelioid variant in order to diagnose the former correctly.

\section{Materials and Methods}

All cases of melanoma diagnosed on cytology from August 2009 to January 2013 were retrieved from our archives. These included a total of 55 aspirates from 45 patients from various sites including lymph nodes, skin nodules, liver, breast, spleen and pancreas. In 6 patients, 2 or 3 synchronous metastatic sites were aspirated. Aspirates were obtained from superficial swellings by cytopathologists using a 22- to 24-gauge needle and from deep-seated masses by radiologists using image guidance. The air-dried smears were stained with May-Grünwald-Giemsa, while the alcohol-fixed smears were stained with the Papanicolaou stain.

The smears were reviewed for cellularity, architecture (cohesion, dyscohesion), cell type, nuclear pleomorphism, nucleolar prominence, intranuclear cytoplasmic inclusions, cytoplasmic vacuoles and melanin pigment. The cases were divided according to the cell type into pure spindle cell type (when more than $90 \%$ of the cell population consisted of spindle-shaped cells), epithelioid cell type (when more than $90 \%$ of the cells were of the epithelioid type) and mixed cell type (when more than $10 \%$ of the cells of either cell type were present). In addition, the histopathology slides were reviewed in cases of spindle and mixed cell-type melanoma.

\section{Results}

The patient population included 24 men and 21 women with a mean age of 46.8 years (range $22-70$ years) and 54.6 years (35-68 years) in the epithelioid and spindle cell groups, respectively. Cytomorphologically, the spindle cell group (including both pure spindle cell and mixed cell types) included 14 (31\%) patients, while the morphology was predominantly epithelioid in 31 (69\%) patients out of a total of 45 cases. The most common sites aspirated in the spindle cell group were lymph nodes (8), liver (3), chest wall and shoulder (1), spleen (1) and pancreas (1). In the cases of epithelioid cell morphology, the aspirates were from lymph nodes (27), thigh (4), arm (3), breast (2), liver (2), presacral region (1), back (1) and foot (1). In all cases melanoma had been diagnosed, and they all underwent aspiration due to clinical suspicion of either recurrence or metastasis. Detailed information about the primary diagnosis, primary site and duration of the interval from diagnosis to recurrence or metastasis could not be obtained due to the retrospective nature of the study except for 2 patients with the spindle cell type where the primary site was biopsied from the foot, and lymph nodes were aspirated due to clinical suspicion of metastasis.

Aspiration smears exhibited moderate to high cellularity in most of the cases in both groups. The spindle cell type demonstrated a greater degree of cellular cohesion (92.8\%) (fig. 1a, b) compared to the epithelioid cell type which showed cellular cohesion (fig. 1c, d) in only $13 \%(4 / 31)$ of the cases $(p<0.0001)$ (table 1$)$. Singly scattered epithelioid cells were found in addition to the cohesive spindled fragments in the mixed-cell-type cases. The spindle cells were characterized by slender spindle-shaped cells with oval to slender nuclei displaying mild to moderate pleomorphism. The epithelioid cells were dispersed and plasmacytoid to polygonal in shape with abundant well-defined cytoplasm. Nucleolar prominence and the presence of macronucleoli was a characteristic feature of epithelioid cells (fig. 1e), whereas spindle cells demonstrated inconspicuous small nucleoli (fig. 1f). There was no significant difference between the 2 groups in the presence of melanin pigment. Intracellular as well as extracellular melanin pigment was found in 78.5 and $80.6 \%$ of cases of spindle cell and epithelioid cell types, respectively. Nuclear pleomorphism varied from mild to moderate in most of the cases of spindle cells (78.5\%) with very few cases exhibiting marked nuclear atypia with the presence of tumor giant cells. In contrast, 
Fig. 1. a, b Cohesive fragments of spindleshaped tumor cells with few scattered dyscohesive cells in the background. c The epithelioid variant shows mainly dispersely distributed polygonal to plasmacytoid cells with occasional cell grouping (d) and prominent macronucleoli (e). f In contrast, spindle cells do not show prominent nucleoli. Papanicolaou. a $\times 40$. e, $\mathbf{f} \times 400$. MayGrünwald-Giemsa. $\mathbf{b} \times 40$. c, $\mathbf{d} \times 200$.

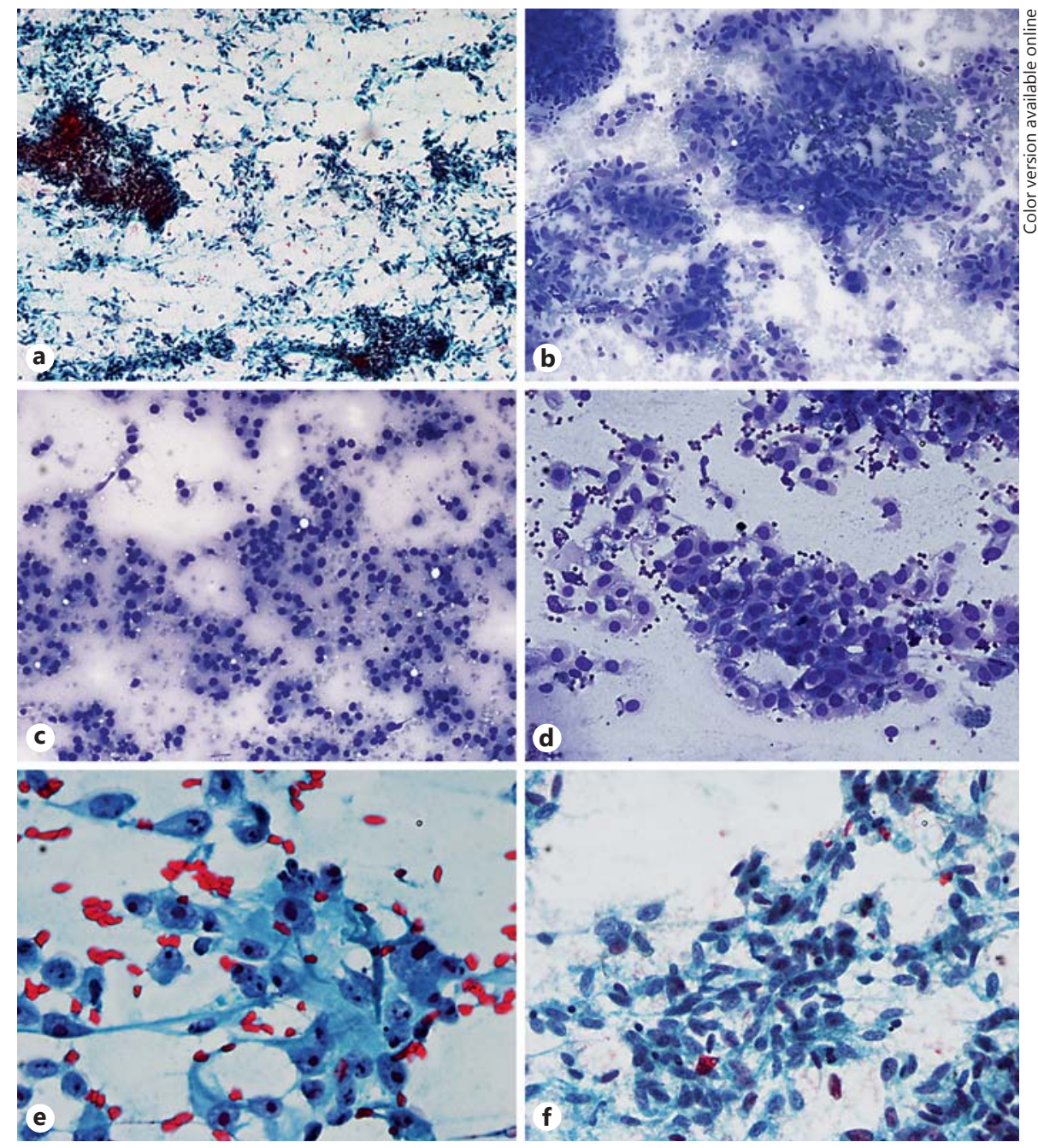

the epithelioid variant showed moderate to marked nuclear atypia (78\%) in most of the cases. Bland nuclear chromatin was found in $22 \%$ of the cases of the epithelioid type. Cytoplasmic vacuoles were not identified as a prominent feature in either of these 2 groups.

Aspirates from different sites of the same patient (6 patients) showed a similar epithelioid type. Immunocytochemistry for HMB- 45 was done in all pure spindle cell melanomas ( 6 cases) and 11 cases [liver (2), breast (2) and lymph nodes (7)] of the epithelioid type for confirmation of the diagnosis (fig. 2a, b) which was positive.

Surgical specimens were available in 10 out of 14 cases of spindle and mixed cell types. Biopsy/excision specimens were from the same site in 2 cases but from a different site in 8 cases. Most of the cases correlated well with regard to the cell type (fig. $3 \mathrm{a}-\mathrm{c}$ ) except in 1 case. In this case the biopsy from the preauricular nodule was com- posed predominantly of epithelioid cells with minimal spindling and prominent nucleoli, whereas aspirates from splenic metastasis exhibited predominant spindle cell morphology with a small amount of epithelioid cells. Smears from 2 cases (from lymph node metastasis) with the absence of melanin pigment revealed the presence of melanin in the biopsy specimen taken from the primary site (foot in both cases). Immunohistochemistry for S-100 and HMB-45 was done in 6 cases for confirmation of the diagnosis which showed positivity in all cases.

Overall, the characteristic cytological features of melanoma (dyscohesive cell population of polygonal to plasmacytoid cells with abundant well-defined cytoplasm and the presence of macronucleoli) are not very frequently observed in spindle cell melanomas and, if present, they are usually confined mostly to the coexisting epithelioid cell population. 
Fig. 2. HMB-45 immunostain shows cytoplasmic positivity in spindle (a) and epithelioid (b) types. a, b Original magnification, $\times 400$.
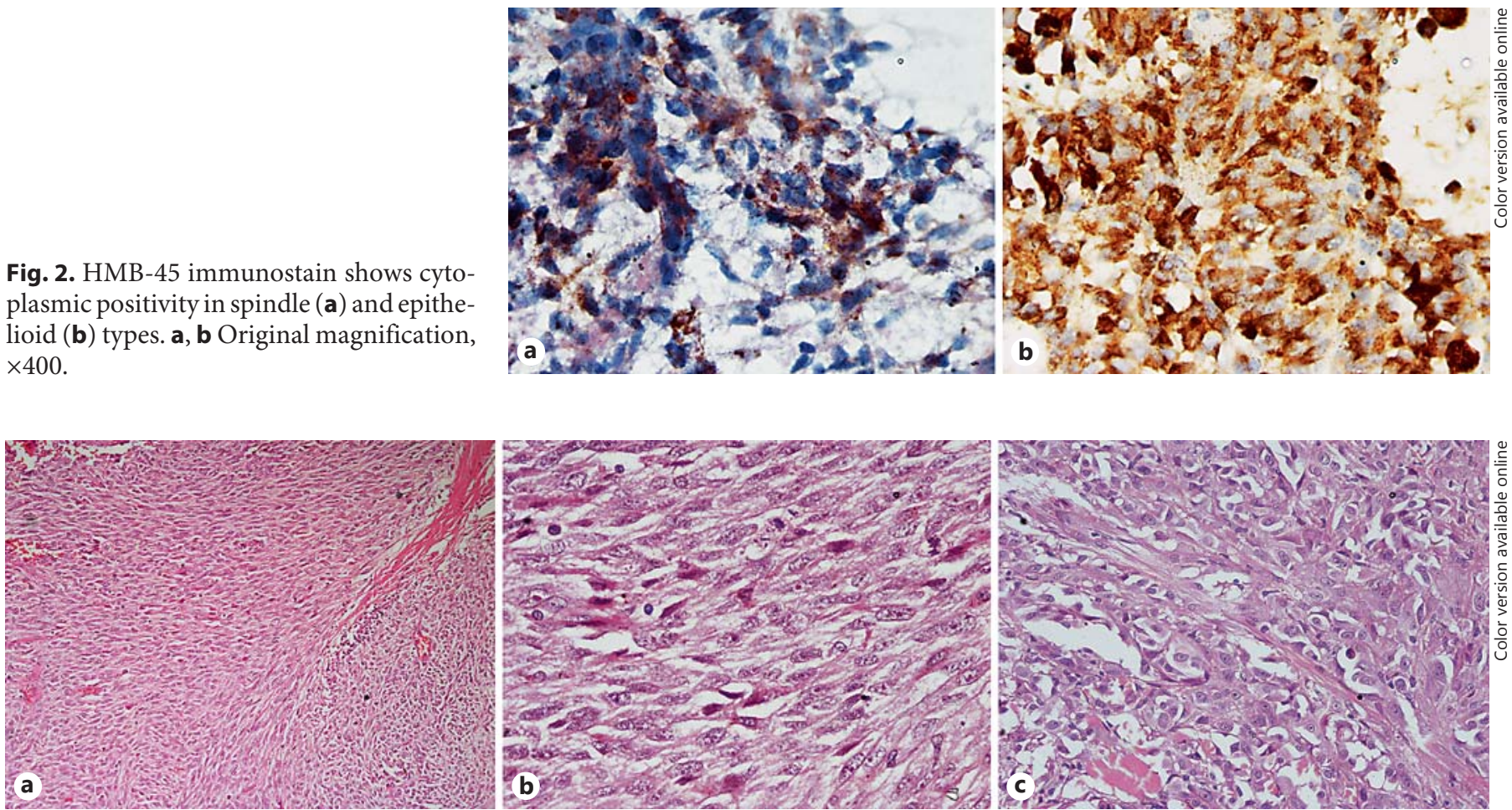

Fig. 3. Biopsy specimens show spindle cells in fascicular pattern $(\mathbf{a}, \mathbf{b})$ and epithelioid cells with macronucleoli $(\mathbf{c})$. HE. $\mathbf{a} \times 100 . \mathbf{b} \times 400$. c $\times 200$.

\section{Discussion}

FNA cytology is a widely accepted diagnostic technique used for early detection of malignancies. Melanomas can be readily diagnosed by cytomorphology alone since most of the cases present with typical features such as dispersed cell population of plasmacytoid to polygonal cells with intracytoplasmic melanin pigment, intranuclear inclusions and the presence of macronucleoli $[7,8]$. However, it is not unusual for this tumor to display divergent cellular morphology which frequently poses a diagnostic problem, especially if the clinical history is not suggestive or immunological markers are not available $[3,5]$. The patterns of melanoma in cytology include the classical, carcinoma-like, spindle cell type, lymphoma-like, myxoid, clear cell and undifferentiated type [6]. Clinically, the spindle cell melanoma and its desmoplastic variant are known to occur at a slightly older age [9] as was evident in our series though not statistically significant possibly due to a smaller number of patients. Lymph nodes are the most common site of metastasis in both groups.

Spindle cell morphology is not infrequently seen in cases of melanomas. We found that $31 \%$ of the cases showed a pure or a mixed spindle cell population. Out of these, $13 \%$ of the cases were composed purely of spindle cells in accordance with the previous reports.

Spindle cell melanomas do not exhibit the classical features of melanoma and hence can be mistaken for a sarcomatous neoplasm when nuclear atypia is present or a benign soft tissue spindle cell tumor if the nuclear morphology is bland $[5,8]$. The presence of intracellular melanin pigment can be used as an important clue to the diagnosis which was present in approximately $80 \%$ of our cases. Recognizable melanin pigment is seen variably in the spindle cell variant $[4,5,7,10-16]$. It becomes more clinically challenging if spindle cells are present at metastatic sites such as lymph nodes, liver and spleen without an obvious clinical history of melanoma. If spindle cells are bland, they may be misdiagnosed as either reactive fibroblasts or centrocytes of reactive lymph nodes; in case of pleomorphic tumor cells, the distinction from sarcoma cells is difficult. In the present series cellular aspirates, variably pleomorphic tumor cells and a background history of melanoma were helpful features for the diagnosis.

Immunocytochemistry for specific melanoma markers can be utilized in amelanotic cases or when other pig- 
mented lesions such as pigmented dermatofibrosarcoma or nerve sheath tumor are considered in the differential diagnosis [5, 17-19].

Cytomorphologically, spindle cell melanomas demonstrated a greater degree of cellular cohesion as compared to the epithelioid type. Although not statistically significant, the absence of significant nuclear pleomorphism and the lack of macronucleoli are other features of spindle cell melanoma in contrast to the epithelioid variant.

Histologically, spindle cell melanoma is a form of vertical growth phase melanoma in which the invasive tumor cells have a spindled morphology and are variably associated with a desmoplastic stromal response. There appears to be a morphological continuum between desmoplastic melanoma and spindle cell melanoma. The neoplastic melanocytes may either acquire a single-cell dispersal pattern within the sclerotic stroma or be disposed in long fascicles similar to spindle cell sarcoma. Reactive fibroblasts are intimately admixed with the tumor cells and are presumed to be the source of matrix production [20,21]. Most of the cases of spindle and mixed cell type in the present series were composed of sheets and fascicles of purely spindle cells or epithelioid cells with spindle cell areas. Desmoplastic collagenized stroma was not seen as a prominent feature in either of these cases. Most of the findings correlated well with the cytological features except in one case where there was a discrepancy in the type of the predominant cell type. Piao et al. [5] in their series found a discrepancy of the cell type on histology in $20 \%$ of the cases.

This study describes the characteristic cytomorphological features of spindle cell melanoma and highlights differences from the epithelioid variant. The prominent nucleoli and cellular dyscohesion, hallmark features of melanoma, do not characterize spindle cell melanomas. However, cellular cohesion and inconspicuous nucleoli typify spindle cell melanomas on cytology. On clinical comparison, a spindle cell variant occurs at a slightly higher age. An awareness of clinical and cytomorphological features greatly improves the diagnostic certainty of spindle cell melanomas on cytology.

\section{Disclosure Statement}

The authors have no conflict of interest to declare.

\section{References}

1 Leong SP, Gershenwald JE, Soong SJ, Schadendorf D, Tarhini AA, Agarwala S, Hauschild A, Soon CW, Daud A, Kashani-Sabet M: Cutaneous melanoma: a model to study cancer metastasis. J Surg Oncol 2011; 103:538-549.

- 2 Schmid-Wendtner MH, Baumert J, Schmidt M, Konz B, Hölzel D, Plewig G, Volkenandt M: Late metastases of cutaneous melanoma: an analysis of 31 patients. J Am Acad Dermatol 2000;43:605-609.

-3 Banerjee SS, Harris M: Morphological and immunophenotypic variations in malignant melanoma. Histopathology 2000;36:387-402.

-4 Saqi A, McGrath CM, Skovronsky D, Yu GH: Cytomorphologic features of fine-needle aspiration of metastatic and recurrent melanoma. Diagn Cytopathol 2002;27:286-290.

5 Piao Y, Guo M, Gong Y: Diagnostic challenges of metastatic spindle cell melanoma on fine needle aspiration specimens. Cancer Cytopathol 2008;114:94-101.

6 Nasiell K, Tani E, Skoog L: Fine needle aspiration cytology and immunocytochemistry of metastatic melanoma. Cytopathology 1991;2: 137-147.

7 Gupta SK, Rajwanshi AK, Das DK: Fine needle aspiration cytology smear patterns of malignant melanoma. Acta Cytol 1985;29:983988.
${ }_{8}$ Murali R, Doubrovsky A, Watson GF, McKenzie PR, Lee CS, McLeod DJ, Uren RF, Stretch JR, Saw RP, Thompson JF, Scolyer RA: Diagnosis of metastatic melanoma by fineneedle biopsy: analysis of 2,204 cases. Am J Clin Pathol 2007;127:385-397.

-9 de Almeida LS, Requena L, Rütten A, Kutzner H, Garbe C, Pestana D, Gomes MM: Desmoplastic malignant melanoma: a clinicopathologic analysis of 113 cases. Am J Dermatopathol 2008;30:207-215.

10 Yamada T, Itou U, Watanabe Y, Oashi S: Cytologic diagnosis of malignant melanoma. Acta Cytol 1972;16:70-76.

-11 Layfield LJ, Ostrzega N: Fine needle aspirate smear morphology in metastatic melanoma. Acta Cytol 1989;33:606-612.

12 Kline TS, Kannan V: Aspiration biopsy cytology and melanoma. Am J Clin Pathol 1982;77: 597-601.

13 Murali R, Loughman NT, McKenzie PR, Watson GF, Thompson JF, Scolyer RA: Cytologic features of metastatic and recurrent melano$\mathrm{ma}$ in patients with primary cutaneous desmoplastic melanoma. Am J Clin Pathol 2008; 130:715-723.
14 Friedman M, Forgione H, Shanbhag V: Needle aspiration of metastatic melanoma. Acta Cytol 1980;24:7-15.

15 Perry MD, Gore M, Seigler HF, Johnston WW: Fine needle aspiration biopsy of metastatic melanoma. A morphologic analysis of 174 cases. Acta Cytol 1986;30:385-396.

16 Woyke S, Domagala W, Czerniak B, Strokowska M: Fine needle aspiration cytology of malignant melanoma of the skin. Acta Cytol 1980;24:529-538.

17 Kapila K, Kharbanda K, Verma K: Cytomorphology of metastatic melanoma - use of S-100 protein in the diagnosis of amelanotic melanoma. Cytopathology 1991;2:229-237.

18 Kobayashi G, Cobb C: A case of amelanotic spindle-cell melanoma presenting as metastases to breast and axillary lymph node: diagnosis by FNA cytology. Diagn Cytopathol 2000; 22:246-249.

19 Marco V, Sirvent J, Alvarez Moro J, Clavel M, Muntal MT, Bauza A: Malignant melanotic schwannoma fine-needle aspiration biopsy findings. Diagn Cytopathol 1998;18:284-286.

20 Bruijn JA, Mihm MC Jr, Barnhill RL: Desmoplastic melanoma. Histopathology 1992;20: 197-205.

21 Magro CM, Crowson AN, Mihm MC: Unusual variants of malignant melanoma. Mod Pathol 2006; 19(suppl 2):S41-S70. 